\title{
Le « reddito di cittadinanza » italien en recherche d'identité : approche européenne et comparative
}

Elena Anna Grasso

\section{(2) OpenEdition}

1 Journals

Édition électronique

URL : https://journals.openedition.org/rdctss/1189

DOI : $10.4000 /$ rdctss. 1189

ISSN : 2262-9815

Éditeur

Centre de droit comparé du travail et de la sécurité sociale

Édition imprimée

Date de publication : 1 avril 2020

Pagination : 66-79

ISSN : 2117-4350

\section{Référence électronique}

Elena Anna Grasso, « Le « reddito di cittadinanza » italien en recherche d'identité : approche

européenne et comparative », Revue de droit comparé du travail et de la sécurité sociale [En ligne], 1 |

2020, mis en ligne le 01 novembre 2021, consulté le 11 novembre 2021. URL : http://

journals.openedition.org/rdctss/1189; DOI : https://doi.org/10.4000/rdctss.1189

\section{cc)}

Revue de droit comparé du travail et de la sécurité sociale est mise à disposition selon les termes de la Licence Creative Commons Attribution - Pas d'Utilisation Commerciale - Pas de Modification 4.0 International. 


\title{
LE « REDDITO DI CITTADINANZA » ITALIEN EN RECHERCHE D'IDENTITÉ : APPROCHE EUROPÉENNE ET COMPARATIVE
}

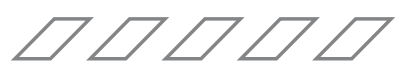

\section{RÉSUMÉ}

Cet article analyse la notion de Reddito di cittadinanza, un instrument social conçu pour lutter contre la pauvreté croissante. Créé par le législateur italien avec la loi n 4 du 28 janvier 2009, le Reddito di cittadinanza vise à garantir une allocation mensuelle pour intégrer les revenus des familles avec des ressources économiques limitées. Malgré la couverture médiatique de ce nouveau dispositif, la réforme contient de nombreuses limites. La présente contribution propose une analyse comparative du dispositif italien avec ceux d'autres États membres européens.

MOTS CLÉS: Aide sociale; sécurité sociale; allocation sociale; revenu de solidarité; allocation chômage.

\begin{abstract}
The article takes into consideration the notion of Reddito di cittadinanza (Italian basic income), a social instrument conceived to combat growing poverty. Established by Italian legislator with law 28 january 2009, no. 4, the Reddito di cittadinanza aims to guarantee a monthly allowance to integrate the incomes of families with limited economic resource. Despite the media emphasis that accompanied the provision, the reform has many limitations. The present contribution analyzes the intersection of the Italian discipline with those of other European Member States.
\end{abstract}

KEY WORDS: Italian welfare; social security; social work allowance; comparative basic income; unemployment allowance. 


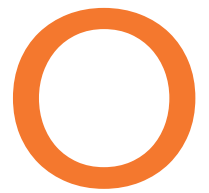

bjet de campagnes électorales de plus en plus houleuses, emblème du retour à la centralité de l'individu, présenté comme une nouvelle institution rompant avec les politiques des gouvernements précédents, le reddito di cittadinanza (RDC) est enfin une réalité.

Toutefois, le battage médiatique qui a accompagné sa mise en œuvre ne correspond pas à une réelle innovation parmi les institutions déjà existantes dans le système de protection sociale italien et, d'une manière générale, au sein de l'Union Européenne.

Largement inspiré par le modèle allemand de l'Arbeitslosengeld II (ou Hartz IV) mis en place en 2005, et récemment critiqué pour la faible mobilité des bénéficiaires sortants ${ }^{1}$, le $\mathrm{RDC}$ est configuré comme une mesure active de politique du travail et de lutte contre la pauvreté, les inégalités et l'exclusion sociale. II consiste principalement en une subvention pour les revenus inférieurs à 500 euros, ainsi qu'en une contribution pour le logement. Pour 2019, le budget alloué au RDC s'élève à 7,1 milliards d'euros², soit un investissement nettement inférieur à celui des mesures similaires en France (10,62 milliards d'euros $)^{3}$ ou en Allemagne (21 milliards d'euros $)^{4}$, d'autant plus si l'on considère que par une disposition législative expresse, une partie considérable du budget ne servira pas à financer la subvention mais à renforcer les agences pour l'emploi et ANPAL Servizi S.p.A., I'organe de l'Agenzia Nazionale per le Politiche Attive del Lavoro (Agence nationale pour les politiques actives pour l'emploi) qui coordonnera les activités prévues par le Patto per il Lavoro (Pacte pour l'emploi) ${ }^{5}$.

Les outils de lutte contre la pauvreté ne sont pas inconnus du législateur italien (I). Comme cela arrive souvent, l'accent mis sur les innovations formelles ne correspond pas à un réel changement de perspective qui, pour être réellement tel, devrait aller de pair avec un budget économique adapté pour soutenir sa structure globale (II).

1 Selon l'association professionnelle Paritätischer Wohlfahrtsverband, qui regroupe les principales associations actives dans le secteur social, 42 \% des chômeurs de longue durée perçoivent la subvention depuis plus de 4 ans, et plus d'un million même depuis son introduction : https://www. der-paritaetische.de/presse/hartz-iv-paritaetischer-fordert-menschenwuerdige-neuausrichtungder-grundsicherung-fuer-arbeitslose-un/

2 Arti. 1, § 255, de la loi nº 145 du 30 décembre 2018 (Loi de finances 2019).

3 Données fournies par la Caisse nationale des allocations familiales : www.caf.fr

4 Données de l'Agence fédérale pour l'emploi, rapportées par «Zeit Online » : https://www.zeit.de/ wirtschaft/2018-10/arbeitslose-hartz-iv-sanktionen-gesunken-leistungskuerzungen-jobcenter

5 L'article 1, § 258, de la loi n 145 du 30 décembre 2018 (Loi de finances 2019) prévoit que « dans le Fonds pour les revenus de la citoyenneté mentionné au paragraphe 255, un montant pouvant atteindre 1 milliard d'euros pour chacune des années 2019 et 2020 est alloué aux agences pour l'emploi en vertu de l'article 18 du décret législatif du 14 septembre 2015, n 150 , en vue de leur renforcement, et un montant maximal de 10 millions d'euros pour l'année 2019 est destiné au financement de la contribution au fonctionnement de l'ANPAL Servizi Spa». 


\section{I - LE REDDITO DI CITTADINANZA, UNE ÉNIÈME MESURE DE LUTTE CONTRE LA PAUVRETÉ}

Le reddito di cittadinanza est une mesure de lutte contre la pauvreté et d'incitation à la recherche d'emploi, introduite en Italie par le décret-loi n 4 du 28 janvier 2019, suivi de la loi n 26 du 28 mars 2019 qui fait suite elle-même à d'autres tentatives législatives (A) et qui se présente comme une solution nouvelle (B).

\section{A - LES PRÉCÉDENTS DU REVENU DE CITOYENNETÉ}

En dépit de son intitulé, la mesure qui était au centre de la campagne électorale du "Mouvement 5 étoiles » aux élections politiques de 2018 et qui constitue l'une des dispositions manifestes du Gouvernement de Giuseppe Conte, doit davantage être considérée comme un revenu d'inclusion que comme un revenu universel ou un revenu versé sans condition à tout un chacun, sur une base individuelle, sans vérification des conditions économiques ou des demandes de disponibilité à travailler. L'Association internationale pour la diffusion du Basic income (véritable revenu universel) définit en effet ce revenu comme " an income unconditionally granted to all on an individual basis, without means-test o work requirement " [un revenu inconditionnellement accordé à tous sur une base individuelle, sans condition de ressources ni d'obligation de travail].

Au-delà du débat scientifique, parfois passionné, qui a animé la réflexion américaine des années soixante ${ }^{6}$, l'application pratique d'une telle forme inconditionnelle de revenu en faveur de chaque citoyen n'est pas très répandue. Le seul modèle d'un revenu de base conceptuellement comparable au reddito di cittadinanza dans sa version authentique est le cas de l'Alaska (USA), où l'Alaska Permanent Fund - le fonds public qui gère les bénéfices de l'exploitation du pétrole - fournit depuis 1982 un dividende monétaire à chaque citoyen. La nature particulière du fonds fait de cette provision une allocation périodique et variable, conçue davantage pour compenser l'exploitation des ressources naturelles que pour garantir un revenu minimum. Même l'ampleur du montant, qui en 2017 a été fixé à environ 1.100 dollars par an et par habitant, éloigne la providence de ce concept de revenu de base suffisant pour garantir le revenu du bénéficiaire.

La mesure introduite en Italie est donc conçue comme un revenu d'inclusion ou un versement d'argent public aux personnes qui se trouvent dans une situation donnée de revenus et de patrimoine et qui acceptent de commencer à travailler ${ }^{7}$. Le reddito di cittadinanza introduit en 2019 n'est pas la première mesure de lutte contre la pauvreté conditionnée par la situation économique du bénéficiaire. La loi n³ 33 du 15 mars 2017

6 J. J. Klos, «Public Assistance, Family Allowance, or the Negative Income Tax » Nebraska Journal of Economics and Business, vol. 8, n² 2, 1969, p. 16; W. D. Popkin, "Administration of a Negative Income Tax ", Yale Law Journal 388, 1969, p. 392; R Perlman, "A Negative Income Tax plan for Maintaining Work Incentives ", The Journal of Human Resources, vol. 3, n 3, 1968, p. 289. Pour une reconstruction du revenu universel dans le système juridique américain, voir E. Mostacci, «Precomprensione ideologica v. analisi fattuale : la parabola del basic income nel contesto nordamericano », Diritto pubblico comparato ed europeo, 2019, p. 1.

7 C. Del Bo, «II reddito di cittadinanza : uno sguardo diacronico sul dibattito e qualche considerazione sulla giustificabilitàa morale », Rivista del Diritto della Sicurezza Sociale, Fascicule 4, 2018, p. 711. 
et le Décret-loi n 147 du 15 septembre 2017 ont en effet introduit dans le droit italien le Reddito di Inclusione (REI) qui, à son tour, a remplacé le Sostegno di Inclusione Attiva (SIA) prévu par le Décret-loi n 5 du 9 février 2012, et repris dans la loi nº 35 du 4 avril 2012.

Les RDC, REl et SIA ont pour but commun la lutte contre la pauvreté - laquelle s'est aggravée en Italie en raison des effets de la crise économique ; la mise à disposition par la fonction publique d'un parcours d'accompagnement personnalisé afin de surmonter les difficultés économiques du bénéficiaire ; le paiement de la prestation par le biais d'une carte de paiement électronique dédiée; et enfin l'identification du foyer comme unité minimale permettant de vérifier le revenu et le capital requis pour accéder à la mesure. Ces trois mesures diffèrent profondément, tant dans l'identification du public bénéficiaire, que dans le montant de l'allocation et dans l'implication massive des agences pour l'emploi développées par le RDC pour faire travailler les bénéficiaires.

Le SIA, né de façon expérimentale en 2012 comme Carta per l'inclusione dans douze grandes villes italiennes, était destiné exclusivement aux familles avec enfants et en situation difficile. En 2016, il est devenu un outil général de lutte contre la pauvreté grâce à l'allocation de 600 millions d'euros ${ }^{8}$. Le SIA permettait de verser une allocation mensuelle variable, d'un minimum de 80 euros à un maximum de 400 euros en fonction du nombre de personnes composant le foyer, pour une durée maximale de douze mois. Pour accéder au SIA, il fallait être de nationalité italienne ou bien un étranger résidant en Italie depuis cinq ans, et ayant des ressources d'une valeur ISEE$^{9}$ ne dépassant pas 3.000 euros.

En 2017, le REI remplace le SIA. L'allocation de 2,1 milliards d'euros permet alors d'augmenter le nombre de bénéficiaires, le montant et la durée de la prestation. Le montant de la subvention passe à $187,50 €$ pour un célibataire, et à $539,80 €$ pour une famille de six personnes. Le système d'un versement sur une carte de paiement électronique dédiée reste inchangé, cette Carta REI pouvant être utilisée pour des achats ou des retraits en espèces avec un seuil mensuel. La durée est fixée à 18 mois, terme au-delà duquel elle peut être prolongée de 12 mois supplémentaires moyennant une suspension de 6 mois. Afin d'augmenter le nombre de bénéficiaires, la valeur ISEE maximale pour chaque foyer est portée à 6.000 euros, mais deux exigences patrimoniales sont conditionnées : la limite de 20.000 euros d'actifs immobiliers, à l'exclusion du premier logement, et la limite de 6.000 euros d'actifs mobiliers. Les familles bénéficiaires sont tenues de respecter le projet personnalisé, élaboré par les services sociaux de la commune de résidence, qui comprend des engagements en matière de formation et/ou de recherche d'emploi, ainsi que la fréquence mensuelle des contacts avec le bureau responsable du projet d'inclusion. En 2018, le public potentiel des bénéficiaires du REl était de 700.000 familles, pour environ 2,5 millions de personnes ${ }^{10}$.

8 Décret interministériel du Ministère du travail et du Ministère de l'économie du 26 mai 2016 (publié dans la Gazzetta Ufficiale n 166 du 18 juillet 2016).

9 Acronyme de Indicatore della Situazione Economica Equivalente (indicateur de la situation économique équivalente), I'ISEE est calculé comme rapport entre l'indicateur de la situation économique (I.S.E.) et un paramètre dérivé d'une échelle d'équivalence prenant en compte la composition du foyer et l'existence de conditions sociales spécifiques.

10 Estimations rapportées dans le rapport technique du décret-loi 147/2017 et du projet de loi de budget 2018 faisant référence à des simulations réalisées sur l'échantillon de déclarations de substitution uniques (DSU) ISEE présentées en 2016, préparées par l'INPS pour le ministère du Travail. 
En 2019, le Reddito di cittadinanza se greffe sur le REI en le remplaçant, permettant d'augmenter le nombre de bénéficiaires et l'ampleur de la mesure, tout en maintenant inchangée l'installation de la mesure de lutte contre la pauvreté.

\section{B - LE DÉCRET-LOI N 4 DU 28 JANVIER 2019 : ENTRE VIEUX PROBLÈMES ET NOUVELLES SOLUTIONS}

Le décret-loi $n^{\circ}$ 4/2019 définit le RDC comme une « mesure unique de lutte contre la pauvreté, les inégalités et l'exclusion sociale, pour garantir le droit au travail ainsi que pour favoriser le droit à l'information, à l'éducation, à la formation, à la culture par le biais de politiques de soutien économique et à l'insertion sociale des personnes menacées de marginalisation dans la société et dans le monde du travail ».

Le reddito di cittadinanza remplace le REl en tant que mesure de lutte contre la pauvreté et entend renforcer les politiques actives du travail par le biais des agences pour l'emploi, qui s'occuperont du Pacte pour l'emploi préparé pour chaque bénéficiaire.

La subvention fournie par l'État, à travers I'Istituto Nazionale della Previdenza Sociale (INPS), se compose de deux éléments cumulatifs : le premier comme soutien au revenu, le second comme soutien au droit au logement. La composante de soutien au revenu peut atteindre un maximum de 500 euros par mois pour un foyer composé d'une seule personne, montant qui passe à 900 euros pour un couple avec deux enfants, et s'élève jusqu'à un maximum de 1.050 euros en présence d'autres membres de la famille. La composante de soutien au droit au logement peut quant à elle atteindre 280 euros par mois pour les familles en location, ou jusqu'à 150 euros par mois pour les familles ayant engagé un crédit pour acheter un logement. La durée maximale de la subvention est fixée à dix-huit mois renouvelables par suspension d'un mois. Le RDC est réservé aux personnes de nationalité italienne ou aux étrangers avec droit de séjour, résidant en Italie depuis au moins dix ans, et dont les deux dernières années ont été consécutives. L'extension de la durée minimale de séjour en Italie pour bénéficier de l'avantage, comme vu précédemment passant de 5 ans pour le REI à 10 ans pour le RDC, vise certainement à limiter le nombre de bénéficiaires étrangers, à tel point qu'en janvier 2019, le ministre du Travail a même suggéré de réserver la mesure aux seuls citoyens italiens ${ }^{11}$.

En ce qui concerne les exigences de capital et de revenu, le foyer doit avoir une valeur ISEE inférieure à 9.360 euros, une valeur des biens immobiliers autre que le lieu d'habitation n'excédant pas 30.000 euros, et une valeur des biens mobiliers n'excédant pas 6.000 euros mais pouvant s'élever jusqu'à 10.000 euros en raison du nombre de membres au sein de la famille ou de la présence de personnes handicapées ${ }^{12}$.

Lors de la conversion du Décret au Parlement, il a été précisé que le patrimoine immobilier pertinent au regard du RDC pouvait se trouver en Italie ou à l'étranger, et que pour que la demande de subvention soit acceptée, les citoyens de pays n'appartenant pas à l'Union Européenne devaient être en mesure de présenter une attestation appropriée, établie par l'autorité compétente de l'État étranger, traduite en italien et légalisée par l'autorité consulaire italienne ${ }^{13}$.

11 Déclarations du Ministre du travail et du développement L. di Maio, 4 janvier 2019.

12 Art. 2, § 1, lettre b), D.L. $n^{\circ} 4 / 2019$.

13 Art. 2, § 1 bis, D.L. 4/2019, introduit par la loi du 29 mars 2019, nº 26. 
On peut aisément imaginer que l'innovation pourrait constituer un obstacle concret à l'achèvement de l'examen préliminaire sur les citoyens étrangers, même si la règle prévoit l'exemption de la certification pour les réfugiés politiques et les citoyens des États pour lesquels il est objectivement impossible de se procurer le document, selon une liste qui sera publiée par le ministère du Travail.

En outre, le RDC ne peut être versé aux familles possédant certains biens considérés comme des biens de luxe : par exemple des bateaux ou bateaux de plaisance, véhicules immatriculés au cours des six derniers mois, véhicules à moteur d'une cylindrée excédant $1600 \mathrm{~cm}^{3}$ ou motocycles d'une cylindrée supérieure à $250 \mathrm{~cm}^{3}$ immatriculés au cours des deux années précédentes ${ }^{14}$. Le bénéficiaire est également tenu d'informer I'INPS dans les quinze jours de tout changement patrimonial entraînant la perte des minima requis. Au cours de la conversion parlementaire, il a également été précisé que la perte des minima requis pouvait survenir même en cas d'achat par succession, de donation ou de gain de sommes dépassant les seuils prévus par la loi ${ }^{15}$.

Le versement de la prestation est subordonné à la disponibilité pour travailler des adultes composant le foyer, ainsi qu'à l'adhésion à un parcours personnalisé pour accompagner la recherche d'un travail et l'inclusion sociale. Ce parcours peut inclure des activités destinées à achever des études et à se recycler professionnellement, ou des services à la communauté, selon un projet défini lors de l'entretien mené dans les agences pour l'emploi, qui doit avoir lieu dans les trente jours suivant l'acceptation de la subvention ${ }^{16}$. Par ailleurs, la loi impose aux agences pour l'emploi de prendre en charge la préparation du parcours individuel et la signature du Pacte pour l'emploi, qui consiste à s'engager à consulter les plateformes d'offres d'emploi, à effectuer une recherche active de travail, à accepter d'être inséré dans des cours de formation, à passer des entretiens et des tests de sélection, à accepter au moins une des trois offres d'emploi adéquates.

La congruence de l'offre sélectionnée par les agences pour l'emploi est définie par le décret-loi $n^{\circ}$ 4/2019 et varie en fonction de la durée d'utilisation de la prestation et du nombre d'offres refusées: dans les douze premiers mois, une offre est ainsi considérée adéquate à moins de 100 kilomètres du domicile (ou 100 minutes en transports en commun) s'il s'agit d'une première offre, ou 200 kilomètres s'il s'agit d'une deuxième offre, ou à n'importe quelle distance s'il s'agit d'une troisième offre. Au-delà des douze mois, une offre est considérée adéquate à moins de 250 kilomètres. En cas de renouvellement de la prestation au-delà de dix-huit mois, l'offre provenant de tout lieu situé sur le territoire italien est considérée comme acceptable. Si la troisième offre n'est pas acceptée, cela entraîne la perte de la subvention ${ }^{17}$. Afin d'assurer le service d'orientation et de recherche active, le ministère du travail a annoncé le recrutement de 3000 consultants appelés "Navigator », coordonnés par l'Agenzia Nazionale per le Politiche Attive del Lavoro (ANPAL), chargés

14 Art. 2, § 1, lettre C), D.L. ${ }^{\circ} 4 / 2019$.

15 Art. 3, § 11, D.L. n 4/2019, modifié par la loi du 29 mars 2019, n²6.

16 Art. 4, D.L. $n^{\circ} 4 / 2019$.

17 Art. 4, § 8, lettre b), numéro 5), D.L. $n^{\circ} 4 / 2019$. 
de fournir des services techniques aux agences pour l'emploi gérées par les régions ${ }^{18}$. Le Pacte pour l'emploi peut être remplacé ou accompagné d'un pacte d'inclusion sociale comprenant le parcours individuel prévu par le REl et élaboré par les services sociaux de la commune de résidence.

Tout comme le REl et le SIA, le RDC est versé via une carte de paiement électronique sur laquelle le montant dû est crédité. En sus des achats, la carte RDC permet d'effectuer des retraits dans la limite de 100 euros par mois et d'effectuer un virement mensuel en faveur du propriétaire ou de l'intermédiaire qui a consenti le prêt pour l'achat du bien immobilier. Par disposition expresse de la loi, la carte RDC ne peut pas être utilisée pour des jeux comportant des gains en espèces ou d'autres avantages. L'obligation de dépenser la somme versée au titre du RDC semble contestable, sinon préjudiciable, à l'autonomie individuelle du bénéficiaire. La disposition prévoit en effet un double contrôle : un premier contrôle mensuel, qui implique la déduction le mois suivant du montant non dépensé, jusqu'à un maximum de 20 \%, et un deuxième contrôle semestriel, qui prévoit la déduction des montants non utilisés à l'exception d'une seule mensualité ${ }^{19}$. En bref, le RDC doit être dépensé en totalité, et plutôt rapidement.

Enfin, les bénéficiaires du RDC sont tenus de fournir des services à la communauté dans les domaines culturel, social, artistique, environnemental, éducatif et de protection des biens communs, services organisés par la commune de résidence, pour un minimum de 8 heures par semaine ${ }^{20}$.

\section{II - LES MESURES DE LUTTE CONTRE LA PAUVRETÉ D'UN POINT DE VUE EUROPÉEN ET COMPARÉ}

La lutte contre la pauvreté et le renforcement des politiques actives en faveur de l'inclusion sociale et de l'emploi sont considérés comme une priorité par les instances de I'Union européenne (A), et en particulier à la suite des effets de la crise économique de la dernière décennie (B).

\section{A - LA LUTTE CONTRE LA PAUVRETÉ DANS L'UNION EUROPÉENNE}

L'Union Européenne s'est limitée à coordonner les politiques nationales en utilisant des recommandations, des lignes directrices et d'autres outils typiques de coordination ouverte. Une première mention du « droit fondamental de la personne à des ressources et à des avantages suffisants pour vivre dans le respect de la dignité humaine " se trouve dans la Résolution contre la pauvreté de 1988, dans laquelle il est expressément fait référence à la nécessité d`établir «un revenu familial minimum garanti comme facteur d'insertion des

18 Les «Navigator » sont des consultants sélectionnés et embauchés par ANPAL Servizi S.p.a., mis à disposition des régions qui gèrent les agences pour l'emploi. L'article 12, paragraphe 3, du D.L. $n^{\circ} 4 / 2019$ modifié par la loi n²6/2019 prévoit l'adoption d'un plan extraordinaire de renforcement des agences pour l'emploi et permet à ANPAL Servizi S.p.A. de sélectionner les compétences professionnelles nécessaires pour organiser la mise en place du RDC en concluant des contrats sous forme de missions collaboratives.

19 Art. 3, § 15, D.L. n 4/2019.

20 Art. 4, § 15, D.L. $n^{\circ} 4 / 2019$. 
citoyens les plus pauvres dans la société $»^{21}$.

Avec la Charte des droits fondamentaux de I'Union Européenne, « le droit à une assistance sociale et à une aide au logement visant à garantir une existence digne à tous ceux qui ne disposent pas de ressources suffisantes » a été reconnu22, selon les méthodes établies par le droit communautaire et les législations nationales, dans le but de lutter contre l'exclusion sociale et la pauvreté. Mais il a fallu attendre la crise économique de la fin du millénaire pour que la question du revenu minimum soit replacée au centre de l'Agenda politique européen.

Dès octobre 2008, le Parlement européen, par la Résolution n 2008/2034²3, avait invité les États membres à promouvoir des politiques actives d'inclusion sociale afin de réguler les "régimes de salaire minimum, les prestations qui y sont liées et une aide sociale qui devrait être facilement accessible » et à imaginer un " plan stratégique de politiques d'intégration active, pour permettre d'échapper à la pauvreté et prévenir l'exclusion sociale ».

La même année, la Commission européenne, avec la Recommandation n²008/867/CE, avait invité les États membres à mettre en place un régime d'aide au revenu conditionné par la « disponibilité active au travail ou à la formation professionnelle pour obtenir un emploi, pour les personnes dont la situation le permet ».

En janvier 2017, le Parlement européen a considéré que «l'Union Européenne se doit d'apporter une réponse rapide et tangible au sentiment de frustration et d'inquiétude qui grandit chez de nombreuses personnes - en raison de l'incertitude des perspectives d'avenir, du chômage, des inégalités croissantes et du manque de possibilités, en particulier pour les jeunes» - et a adopté la Résolution n² 2095 par laquelle il a souligné «l'importance de régimes de revenu adéquat minimal pour protéger la dignité humaine et combattre la pauvreté et l'exclusion sociale, ainsi que leur rôle en tant que forme d'investissement social permettant aux citoyens de participer à la société et de poursuivre une formation et/ou de chercher un emploi »24.

Au-delà de cette approche du droit communautaire, tous les pays européens se sont dotés - ou ont renforcé - ces dernières années des instruments de lutte contre la pauvreté. En 2007, seuls trois pays membres n'avaient introduit aucune forme de revenu minimum : la Grèce, la Hongrie et I'Italie, qui était le dernier État membre de l'UE à se conformer à l'orientation communautaire en introduisant le revenu d'inclusion avec le décret législatif 15 septembre $2017 n^{\circ} 147$, entré en vigueur le $1^{\text {er janvier de l'année suivante }}{ }^{25}$.

21 Résolution concernant la lutte contre la pauvreté dans la Communauté européenne du 16 septembre 1988, A2-171/88, §12.

22 Charte des droits fondamentaux de l'Union européenne, art. $34 \S 3$.

23 2008/867 / CE - Recommandation de la Commission du 3 octobre 2008 sur l'inclusion active des personnes exclues du marché du travail [notifiée sous le numéro C (2008) 5737].

24 Résolution du Parlement européen du 19 janvier 2017 sur un pilier européen des droits sociaux (2016/2095-INI).

25 A. Somma, "Contrasto della povertà e politiche attive del lavoro : reddito di cittadinanza, reddito mimino garantito e regime delle condizionalità ", Diritto Pubblico Comparata Europeo, n², 2019, p. 433. 


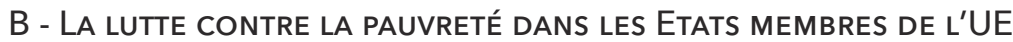

En France, le Revenu de Solidarité Active (RSA), lancé de manière expérimentale en 2005 et étendu à tout le pays en 2009, est une subvention qui propose trois objectifs : intégrer les revenus des familles disposant de ressources économiques limitées, garantir à chacun un revenu minimum qui augmente même si le travail s'accroît à son tour, et disposer d'un soutien social professionnel visant à faciliter la réintégration permanente sur le marché du travail ${ }^{26}$. Le RSA, qui a également une fonction d'intégration pour les bas revenus, s'applique à un public plus large de bénéficiaires. Bien que le calcul du revenu audelà duquel l'attribution peut se faire reste complexe, pour toute une série de coefficients personnalisés, pour un foyer composé d'une seule personne, cette limite est fixée, à titre indicatif, à 14.520 euros par an, contre 9.360 euros prévus par la loi italienne. De plus, en France, la personne qui refuse la deuxième offre de travail perd le droit à la prestation, tandis qu'en Italie, comme évoqué ci-avant, ce droit se perd au troisième refus. En 2017, le Revenu de Solidarité Active a été versé à 1,83 millions de familles résidantes en France métropolitaine ou dans les Départements et Régions d'Outre-Mer, pour un montant moyen de 492 euros par mois et une dépense totale de 10,62 milliards d'euros ${ }^{27}$.

Au Royaume-Uni, le Social Security Contributions and Benefits Act a introduit depuis 1992 l'Income Support, une mesure résiduelle qui ne s'applique que si les autres formes d'assistance, telles que les allocations de chômage, ne peuvent pas être utilisées. La mesure a été remplacée à partir de 2013 par l'Universal Credit, une prestation destinée aux personnes sans emploi et à la recherche d'un emploi, ou dont le revenu est inférieur au minimum légal ${ }^{28}$. Un accompagnement des bénéficiaires dans la recherche d'un emploi par la participation à des entretiens et à des formations est envisagé. Le montant mensuel standard pour un foyer composé d'une personne seule est de 231,67 Livres Sterling, plus une composante pour le droit au logement, qui varie en fonction des circonstances. Les critiques formulées contre l'Universal Credit incluent celles relatives aux procédures de demande de service, qui ne peuvent être effectuées que par voie électronique via le site Web ou l'application du ministère britannique du Travail. Cette méthode risque d'exclure les sujets en marge du numérique, qui sont souvent les plus fragiles socialement et qui, en général, auraient droit à la subvention ${ }^{29}$.

$26 \mathrm{M}$. Borgetto et al., Les débats sur l'accès aux droits sociaux entre lutte contre les exclusions et modernisation administrative, Dossier d'Étude, Cnaf 60/2004; E. Maurel, " Le non-recours aux prestations sociales : les enjeux révélés par le vécu des usagers ", Revue de droit sanitaire et social, 2012, p. 622. Voir également Revue de droit sanitaire et social, $\mathrm{n}^{\circ} 4$ (spécial) dédié au non-recours aux droits, 2012, p. 599, et en particulier C. Willmann, « La renonciation du chômeur : entre tolérance/ignorance et interdiction/sanction», p. 657. Voir également C. Willmann, «Pauvreté laborieuse : la prime d'activité, une bonne réponse? », Droit social, 2015, p. 903.

27 Données fournies par la Caisse Nationale des allocations familiales : www.caf.fr

28 J. O. Frosini, «Dalle Poor Laws all'Universal Credit : lo sviluppo del reddito di base nel contesto britannico ", Diritto Pubblico Comparato ed Europeo, n², 2019, p. 501.

29 V. M. Faioli «I poveri digitali : a proposito di Universal Credit in UK», 2015 : https://www.linkedin. com/pulse/i-poveri-digitali-proposito-di-universal-credit-uk-michele-faioli 
En Allemagne, depuis 2005, l'Arbeistdlosengeld II garantit aux chômeurs n'ayant pas droit aux allocations un chèque mensuel et un parcours d'accompagnement au travail géré par des Jobcenter ${ }^{30}$. La subvention est également accordée aux travailleurs dont le revenu est inférieur à la limite de 9.000 euros. En 2019, le montant de la subvention standard pour un foyer composé d'une seule personne s'élève à 424 euros, montant auquel une composante variable peut être ajoutée pour le droit à un logement jugé convenable pour la famille. En 2017, l'Arbeitslosengeld Il a été versé à 4,08 millions de personnes et a coûté 21 milliards d'euros ${ }^{31}$.

Le modèle allemand, dont le législateur italien s'est largement inspiré32 à la fois pour la centralité des Jobcenter (en Italie les centri per l'impiego) dans la définition du parcours individuel du bénéficiaire, mais aussi pour le système des trois offres de travail, fait l'objet de profondes critiques sur les conséquences à long terme de la mesure ${ }^{33}$. Ceux qui bénéficient de l'Arbeitslosengeld II doivent démontrer leur volonté de chercher un emploi et ne peuvent refuser un emploi raisonnable sous peine de réduire l'allocation. La législation précise que " tout emploi » doit être considéré comme raisonnable ${ }^{34}$, à moins que le sujet ne soit psychologiquement ou physiquement incapable de l'exécuter. En particulier, les professions qui ne correspondent pas à la formation professionnelle ou à l'activité professionnelle précédemment exercée, ou qui présentent des conditions de travail plus défavorables ou sont en quelque sorte inférieures à la qualification professionnelle des chômeurs, sont également considérées comme raisonnables ${ }^{35}$. En effet, le destinataire inactif du service est obligé d'accepter toute offre d'emploi, même sans relation avec la formation et l'expérience professionnelle antérieures. Si le système a permis de ramener le taux de chômage de 11,7 \% en 2005 à 5,2 \% en 2018, il a également favorisé la propagation de l'écart des inégalités, contribuant à accroître la précarité des travailleurs contraints de demander la subvention parce qu'obligés d'accepter des emplois sous-payés. Par cet aspect, mais pas uniquement, la subvention a, durant des années, fait l'objet de discussions controversées. Conçu comme un outil facilitant une réintégration rapide dans le monde du travail, l'Arbeitslosengeld ll est accusé par les critiques d'être devenu un monstre bureaucratique et d'avoir favorisé la précarité. Ceux qui reçoivent la subvention ne sont pas tous au chômage. Parmi eux, il existe aussi des personnes qui ont un emploi,

30 S. Haberl, "Reddito di cittadinanza e reddito minimo garantito nei sistemi tedesco e austriaco», Diritto Pubblico Comparato ed Europeo, n², 2019, p. 534.

31 Données de l'Agence fédérale pour l'emploi, rapportées par Zeit Online : https://www.zeit.de/ wirtschaft/2018-10/arbeitslose-hartz-iv-sanktionen-gesunken-leistungskuerzungen-jobcenter

32 Le phénomène est attribuable à la circulation d'institutions juridiques typiques de la « crossfertilisation " qui consiste à développer les aspects particuliers des droits nationaux résultant d'une sollicitation externe. Voir J. Bell, « Mechanism for Cross-fertilisation od Administrative Law in Europe ", in J. Beatson et T. Tridimas, New Directions in European Public Law, Hart Publishing, Oxford, 1998, p. 147.

33 Selon l'association professionnelle Paritätischer Wohlfahrtsverband, qui regroupe les principales associations actives dans le secteur social, 42 \% des chômeurs de longue durée perçoivent la subvention depuis plus de quatre ans et plus d'un million depuis son introduction : https://www. der-paritaetische.de/presse/hartz-iv-paritaetischer-fordert-menschenwuerdige-neuausrichtungder-grundsicherung-fuer-arbeitslose-un/

34 "Jede arbeit », §10, Sozialgesetzbuch Sgb II.

35 Ibid., §10, alinéa 2. 
mais gagnent trop peu, de sorte qu'ils obtiennent une intégration de la part du Jobcenter. Ce sont les Aufstocker, qui incluent ceux qui, par exemple, effectuent un travail garantissant un salaire maximum de 450 euros par mois, mais aussi de nombreuses personnes qui ont un emploi régulier à temps plein. Depuis l'introduction du salaire minimum en 2015, leur nombre est descendu à sept mille. Au total, les Aufstocker représentent environ 1,2 million de personnes, soit le quart du nombre total de bénéficiaires de la subvention. La somme que l'État leur verse augmente : en 2017, elle a dépassé 10 milliards d'euros ${ }^{36}$.

En Autriche, un accord de 2010 entre l'État fédéral et les Länder a rationalisé et standardisé l'ancien système d'aide sociale, introduisant la "Bms » (Bedarfsorientierte Mindestsicherung), c'est à dire une garantie sociale minimale pour les besoins ${ }^{37}$. II s'agit du troisième et résiduel pilier sur lequel repose l'aide sociale autrichienne, qui s'ajoute à l'Arbeitslosengeld (l'allocation de chômage ${ }^{38}$ ) et au Notstandshilfe (l'aide d'urgence ${ }^{39}$ ). Le "Bms » est une subvention destinée à ceux qui ne sont pas en mesure de subvenir de façon autonome aux besoins nécessaires, et elle est accordée à ceux qui n'ont pas d'emploi ou qui, tout en travaillant, reçoivent des revenus insuffisants pour leur subsistance. La condition de paiement réside dans la situation de nécessité, pour laquelle des limites de revenu et de propriété de la famille sont fixées, le dépassement de ces dernières empêchant de recevoir la subvention. Comme pour l'autre prestation allemande, la «Bms » est détachée de toute référence au dernier revenu et consiste en une allocation mensuelle de montant variable selon le Land, composée d'une somme de base et d'une contribution aux frais de logement. À Vienne, où vivent plus de $60 \%$ des bénéficiaires, la prestation pour 2018 s'élève à un total de $863 €$ par mois ${ }^{40}$.

A l'inverse, le Portugal n'a introduit aucune forme de revenu de citoyenneté, mais a pris des mesures concernant le revenu minimum garanti ${ }^{41}$. Une première mesure appelée Rmg (Rendimento minimo garantido) a été lancée avec la Lei n 19-A du 29 juin 1996 ; elle consistait en une prestation financière de montant variable et de nature temporaire, dans le but de satisfaire les besoins essentiels des personnes et de leurs familles en vue $d^{\prime} u n e$ insertion sociale et professionnelle progressive ${ }^{42}$. Les destinataires étaient des adultes portugais ou étrangers résidant régulièrement dans des conditions économiques et sociales précaires, à qui il a été demandé d'accepter l'emploi qui leur avait été proposé ou de participer à des activités de formation ou d'inclusion sociale. En 2003, la Lei 13 du

36 Données de l'Agence fédérale pour l'emploi : https://www.arbeitsagentur.de/

37 S. Haberl, "Reddito di cittadinanza e reddito minimo garantito nei sistemi tedesco e austriaco », Diritto Pubblico Comparato ed Europeo, vol. 2, 2019, p. 541.

38 L'Arbeitslosengeld dure entre 20 et 52 semaines, extensible jusqu'à 78 semaines, et le montant est fixé à $55 \%$ du dernier salaire.

39 La Notstandshilfe versée durant 52 semaines représente environ 90 \% des allocations de chômage. Elle s'applique uniquement aux chômeurs qui ne sont pas en mesure de satisfaire aux besoins nécessaires de la vie, et prend en compte des conditions économiques, patrimoniales et familiales.

40 https://www.wien.gv.at/statistik/soziales/tabellen/mindestsicherung-erloese.html

41 P. Addis, "Reddito di cittadinanza e reddito minimo garantito in Portogallo e Spagna», Diritto Pubblico Comparato ed Europeo, vol. 2, 2019, p. 462.

42 Art. 1, Lei n 19-A/1996 du 29 juin 1996. 
21 mai 2003 a remplacé le Rmg par le Rsi (Rendimento social de inserção). La mesure - toujours en vigueur aujourd'hui -, bien que reformulée et réduite suite à la crise économique, a prévu des formes de contrôle plus strictes pour éviter les utilisations frauduleuses, et une série d'obligations pour le destinataire, contenues dans le contrat d'inclusion. Le montant maximum de la mesure est fixé à 189,66 € par mois, montant qui peut être majoré sur la base d'éventuels membres supplémentaires de la famille, à hauteur de 132,76€ si le membre familial est âgé de plus de 18 ans et de 96,83 € en-deçà.

En Espagne, il existe une mosaïque complexe de mesures de soutien du revenu, en raison notamment du fait que la Constitution de 1978 délègue l'assistance sociale aux Communautés autonomes ${ }^{43}$, tout en réservant la législation sur la sécurité sociale à la juridiction nationale ${ }^{44}$. Le long de la frontière glissante entre assistance et sécurité sociale, la production législative de l'État central et des Communautés autonomes en matière de revenu minimum s'est développée ${ }^{45}$. Ainsi, le Real decreto n 1369 du 24 novembre 2006 introduit la Rai (Renta activa de inserción) dans tout le pays. Il s'agit d'une mesure destinée aux moins de 65 ans, au chômage et en situation de besoin économique, qui n'ont droit à aucune autre prestation sociale. Les bénéficiaires doivent signer un Compromiso de actividad, participer à des activités de formation et accepter les offres d'emploi qui leur seront présentées. La subvention ne dure pas plus de 11 mois et est fixée pour 2019 à 430 euros mensuels ${ }^{46}$.

Parallèlement à la Rai, qui est de nature essentiellement résiduelle, chaque Communauté autonome a mis en place son propre service de soutien au revenu, libellé différemment et avec des exigences diverses en termes de durée et de portée économique. Les prestations régionales ont en commun l'objectif de garantir un revenu minimum, et de promouvoir le travail et l'inclusion sociale. La durée varie de 6 mois pour l'Ingreso mínimo de solidaridad, à 12 mois pour la Renta mínima de inserción social en Andalousie, à 36 mois pour la Renta valenciana de inclusio en vigueur dans la Comunidad Valenciana, voire jusqu'à une durée illimitée pour la Renta mínima de inserción à Madrid le Salario social básico des Asturies et la Renta garantizada de ciudadanía de Castille-et-León. La mesure de la prestation est comprise entre 300 euros mensuels (pour la Renta básica de inserción de la Région de Murcie) et 665 euros mensuels (pour la Renta de garantía de ingresos du Pays basque). L'ensemble de ces mesures constitue un cadre qui est tout sauf uniforme et en constante évolution, ce qui rend souvent difficile l'accès aux services, en raison du conflit entre les réglementations nationales et les mesures mises en œuvre par les différentes Communautés autonomes ${ }^{47}$.

43 Constitution espagnole, art. 148, point 20.

44 Constitution espagnole, art. 149, point 17.

45 P. Addis, "Reddito di cittadinanza e reddito minimo garantito in Portogallo e Spagna ", Diritto Pubblico Comparato ed Europeo, vol. 2, 2019, p. 467.

46 Voir SEPE, Servicio Publico de Empleo Estatal : www.sepe.es

47 Voir J. Cata, «La inestabilidad pone en jacque la renta garantizada », El Pais, 4.11.2018. 


\section{Conclusion}

Le RDC est la plus grande mesure de lutte contre la pauvreté et de relance de l'emploi mise en place jusqu'à aujourd'hui en Italie. L'investissement alloué par le Gouvernement s'élève à 7,1 milliards d'euros pour 2019, 8 milliards pour 2020 et 8,3 milliards par an à partir de $2021^{48}$. Selon les prévisions du ministère du travail, le RDC aurait dû concerner 5 millions de personnes ${ }^{49}$, doublant le nombre de personnes bénéficiaires du REI. Mais dans les faits et selon les données de l'Observatoire statistique de l'INPS, suite aux demandes présentées par 1,5 million de familles, le RDC n'a été accordé qu'à 982.000 familles et a concerné un nombre de salariés estimé à 2,2 millions, pour un montant mensuel moyen de 482 euros $^{50}$.

Le grand écart entre les prévisions et les premières données dont nous disposons est significatif et témoigne de la difficulté du législateur à construire des mesures efficaces de lutte contre la pauvreté. Malgré les limites décrites ci-dessus, I'Italie s'est dotée, avec le RDC, d'un instrument d'inclusion sociale et professionnelle comparable - au moins dans les montants - aux autres grands pays européens dans lesquels, des institutions similaires fonctionnent depuis plus d'une décennie. Par-delà certaines particularités italiennes - telles que l'interdiction d'utiliser l'allocation à des jeux et l'obligation de la dépenser, le véritable défi pour le RDC comme pour les dispositifs européens semblables réside dans la capacité effective à faire réellement sortir une partie des bénéficiaires de leur situation précaire, et donc à ne pas se transformer en une simple mesure d'assistance.

L'ANPAL et les agences pour l'emploi joueront un rôle central pour relever ce défi, poursuivant l'objectif de renforcer le dispositif d'aide, de réformer le système, tout en suivant le parcours individuel de millions de bénéficiaires du RDC. En 2019, l'action favorisant le retour au travail est apparue encore en phase de démarrage : le recrutement de consultants ANPAL n'est pas terminé - le nombre de "Navigator » ayant fait l'objet d'accords spécifiques entre le Gouvernement et les régions - ; la plateforme télématique est toujours en phase de test et devrait regrouper les demandes et les offres d'emploi, en accordant aux employeurs qui embauchent des bénéficiaires de la RDC un allégement fiscal spécial ${ }^{51}$.

S'il est prématuré de porter un jugement sur l'expérience italienne, les expériences française et allemande, finançant des montants plus importants que le RDC, révèlent à cet égard l'aspect le plus problématique dont la réforme italienne ne tient pourtant pas compte : le risque réel de créer un segment de la population qui subvient de façon quasi permanente à ses besoins grâce à la mesure de soutien au revenu.

48 Le paragraphe 255 de l'article 1 de la loi de finances 2019 prévoit : " Dans les estimations du ministère du travail et des Politiques sociales, un fonds appelé «Fondo per il reddito di cittadinanza » est créé, doté d'un budget de 7.100 millions d'euros pour 2019 et de 8,055 millions d'euros pour l'année 2020 et 8,317 millions d'euros par an à partir de 2021 ».

49 Présentation du Reddito di cittadinanza : http://www.governo.it/sites/governo.it/files/RDC-Q100 1. pdf

50 Observatoire statistique INPS, publié le 22.10 .2019 : www.inps.it/nuovoportaleinps/default. aspx?itemdir $=53155$

51 Ce n'est que le 15 novembre 2019 que l'INPS a publié la procédure informatique pour demander I'incitation au recrutement des personnes bénéficiant du Reditto di cittadinanza. 


\section{LE REVENU DE CITOYENNETÉ ITALIEN}

La solution française qui, grâce à des déductions fiscales spécifiques, permet dans une certaine mesure de ne pas réduire l'indemnisation versée - même si le revenu du travail du bénéficiaire augmente - peut être une incitation à chercher un revenu autre qu'une subvention, mais cela représente certainement une dépense plus lourde pour l'État.

L'Italie n'en est qu'à ses débuts et un suivi attentif de la répartition sociale et territoriale des subventions sera nécessaire, tout comme une analyse du nombre de sanctions, de la fréquence des demandes de renouvellement et de la mobilité des bénéficiaires, afin de vérifier les objectifs atteints par le RDC.

\section{ELENA ANNA GRASSO}

Chercheuse en droit comparé, Université de Gênes.

Thèmes de recherche: Droit de la consommation, droit de la santé, droits linguistiques, droits sociaux.

\section{Publications:}

E. A. Grasso, "The social role of healthcare system. A comparative analysis of case-law regarding patients' right to mobility within the European Union », DPCE Online, 2019.

E. A. Grasso et L. Bairati, « Lire ou regarder ? Les couleurs dans l'étiquetage alimentaire et I'information du consommateur », LexisNexis, n 10, Octobre 2019. 